\title{
From Initial Encounter with Mid-Air Haptic Feedback to Repeated Use: the Role of the Novelty Effect in User Experience
}

\author{
Isa Rutten, Lawrence Van den Bogaert, and David Geerts
}

\begin{abstract}
Mid-air haptic (MAH) feedback is an interesting means to provide augmented haptic feedback for gesture-based technology as it enables a sense of touch without physical contact with an actuator. Although quite some work already investigated the user experience (UX) of MAH feedback during initial encounter, we are not aware of studies testing the UX after repeated use, with regard to both pragmatic and hedonic UX, as well as emotional reactions. In this study, we tested how the UX of MAH feedback changed over the course of five weeks by collecting both questionnaire as well as interview data of 31 participants. Our results showed that MAH feedback significantly increased the enjoyment, engagement, valence and arousal of the emotional response. However, the added value of valence was due to a novelty effect as it was only significantly elevated during initial use, and not after repeated use. Interestingly, the added value of MAH feedback in terms of enjoyment, engagement and arousal remained elevated over the course of five weeks. Moreover, the interview data hinted at substantial individual differences underlying the global trends from the questionnaire data, showing the importance of combining quantitative and qualitative data when testing the UX of MAH feedback.
\end{abstract}

Index Terms-Mid-air haptic feedback, novelty effect, user experience, emotional reaction, repeated use.

\section{INTRODUCTION}

$\mathbf{S}$ YSTEM feedback, in particular haptic feedback, is important to the user as it diminishes uncertainty, and increases a sense of control while interacting with a system or device, making it appear more responsive [1][2]. When considering a gesture-based interface, where intrinsic haptic feedback is absent (e.g. touching a computer keyboard or mouse), augmented haptic feedback, such as mid-air haptic or vibrotactile feedback, plays an important role in improving the user experience. For example: it has been found to increase a sense of control and leads to benefits in terms of task performance, subjective workload, pleasure, attractiveness of the device, and more [1][3][4][5]. One example of augmented haptic feedback is mid-air haptic (MAH) feedback, involving tactile sensations rendered in mid-air without physically touching an actuator. These tactile sensations are produced through ultrasound [6] or air vortices [7]. Especially in combination with a gesture-based interface, MAHs are a promising haptic feedback modality because they have the advantage of enabling spontaneous interaction as no actuator needs to be attached to the hand or arm [3][8][2]. Another example of augmented haptic feedback

All authors are from the Meaningful Interactions lab (Mintlab), KU Leuven, Belgium. Corresponding author: David Geerts (david.geerts@kuleuven.be) is contact-based vibrotactile feedback, with the advantage of enabling concise and salient sensations [2], but the disadvantage of having an actuator attached to user's hand or arm.

Thus, MAH feedback, although delivering a more subtle sensation compared to contact-based haptic feedback [2], is the preferred modality when spontaneous and unhindered gestures are prioritized, such as when interacting with digital kiosks at public spaces, or during gesture-based interaction in cars [9][3]. However, MAH feedback is a relatively novel technology and to our knowledge not yet implemented in computers or smartphones (or any type of smart device). A substantial body of literature is already available about the way MAHs add to the user experience in a variety of contexts. For example, MAH feedback during gesture-based interaction has shown to reduce visual demand and increase accuracy in slider-bar tasks in an automotive context. In a more abstract gesture-based interface, MAH feedback has also shown hedonic UX benefits: it increased attractiveness and experienced pleasure during interaction [5]. Moreover, MAHs have shown to increase the user's engagement during gesturebased interaction with digital kiosks [9].

However, while the novelty effect is an important challenge that needs to be overcome when assessing the application potential of a new technology [10], little research has investigated how the novelty of this technology could potentially impact the user experience. There are two exceptions where the impact of perceived novelty was either explicitly addressed [5], or where the user experience of MAHs was assessed at two points in time [11]. In a recent study [5], the added value of MAHs in terms of increased attractiveness and experienced pleasure in a gesture-based interface disappeared after statistically controlling for perceived novelty. In the study by Ablart et al. [11], MAH feedback while watching a movie led to higher self-reported arousal both during participants' first and second experience with MAHs. Based on these two studies, it appears that certain aspects of the user experience could be more sensitive to a novelty effect (e.g. attractiveness, pleasure), while self-reported arousal appeared to be rather unaffected. However, these studies are not directly comparable as the first one describes a cross-sectional approach and the second a longitudinal approach. Moreover, in the first study [5] perceived novelty was explicitly assessed, whereas in the second [11] there was no explicit operationalization of the novelty effect.

Interestingly, a golden standard of how to define or assess the novelty effect is missing. Koch et al. [10] combined 
definitions from a variety of sources, summarizing the novelty effect in the context of HCI as: "an increased motivation to use something, or an increase in the perceived usability of something, on account of its newness. When novelty eventually fades, usage patterns and/or perceived usability changes" (p. 3) [10]. Although this definition focuses on usage patterns and usability, it is important not to forget about other qualities of the user experience as well and how novelty can impact those. Indeed, apart from usability or pragmatic UX, hedonic qualities of the UX matter too [12][13], as well as users' emotional reactions [14]. Apart from the lack of a comprehensive definition of the novelty effect, there is no consensus either about how to assess it, such as how to identify the presence of a novelty effect or how to understand the temporality of the novelty effect (such as after how many weeks the novelty effect can be expected to wear off).

The contribution of this paper is twofold. First, we describe the impact of novelty on the UX of MAHs by comparing the UX during initial encounter, and after repeated use. Second, we report whether the presence of a novelty effect could be identified based on a questionnaire variable: perceived novelty. To this end, we studied how perceived novelty evolved over time, and described whether or not this pattern was in line with what we expected from the literature. As MAHs are not yet implemented in everyday technology, this longitudinal study was conducted in-lab, where participants could experience MAHs in the context of a gesture-based home automation system. We chose the context of a home automation system because it enabled us to explore the value of MAHs when added to diverse gestures and functions. The study was run over the course of five weeks, involving eight different sessions per participant, with a total of 31 participants. We administered questionnaires during the first and the last session, to assess perceived novelty, pragmatic and hedonic UX qualities, and users' emotional reactions. Participants filled out these questionnaires four times in total: twice in session 1 and twice in session 8. In both sessions, one questionnaire asked about their experience with MAHs, and one questionnaire about their experience without MAHs. This enabled us to calculate the difference score to assess the added value of MAHs. Furthermore, we also collected qualitative data in the form of open-ended questions about participants' preferences regarding the addition of MAHs to the gesture-based system.

Our results showed an added value of MAHs in terms of enjoyment (hedonic UX), engagement, valence and arousal of users' emotional response. A novelty effect appeared to be present for emotional valence, but not for enjoyment, engagement, or arousal. Users' enjoyment when interacting with the device was lower after repeated use, but the added value of MAH feedback in terms of enjoyment did not disappear after repeated use. Therefore we would conclude that the increased enjoyment when MAH feedback was present, was not due to a novelty effect. Moreover, the device with MAH sensations was perceived as more hedonic than without MAH feedback both during initial and after repeated use. Regarding pragmatic UX, we observed no added value of MAHs. Perceived novelty was significantly higher for the system with MAHs both during initial use and after repeated use, and there was no decrease in perceived novelty over the five week period. Finally, the interview data showed interesting individual differences underlying the general trends observed in the questionnaire data.

\section{RELATED WORK}

\section{A. The User Experience of MAH Feedback}

Following the CUE model (Components of User Experience) [14], one can differentiate between three crucial UX components, forming together a holistic model of UX: instrumental qualities, non-instrumental qualities, and emotional reactions. Instrumental qualities refer to usability and usefulness, which can be understood as pragmatic UX, whereas non-instrumental qualities refer to appeal and attractiveness or hedonic UX. The emotional component involves subjective feelings, but also physiological reactions, and more [14]. Similarly, Maggioni et al. [15] studied the added value of MAH feedback by assessing pragmatic and hedonic UX qualities, users' emotional reactions (valence and arousal) and expectations. When considering the technology acceptance model (TAM [16], and more recent developments based on this prominent model (e.g. the unified theory of acceptance and use of technology (UTAUT [17]), both pragmatic (performance and effort expectancy) and hedonic (enjoyment) qualities of the UX predict intention to use and continued use. However, depending on whether users perceive the system as predominantly utilitarian versus hedonic, the predictive strength of the pragmatic versus hedonic aspects changes [17] [18]. The more users perceive the system to be fun-oriented (and less utility oriented), the more enjoyment predicts intention to use and continued use, instead of usefulness [18] [19]. Although pragmatic and hedonic UX is often assessed separately, it can also be combined into one construct: user engagement. User engagement is described as a UX quality involving the depth of the user's cognitive, temporal, affective and behavioral investment during interaction. Engagement consists of items that assess aesthetic appeal, focused attention, perceived usability, and reward [20][21]. A recent study observed evidence for significantly higher engagement with digital kiosks when MAH feedback was present versus absent [9]. Apart from this exception, where pragmatic and hedonic UX are combined into one construct (engagement), more often pragmatic and hedonic UX are assessed as separate constructs. Therefore, in the remainder of this related work section, we divide the pragmatic and hedonic UX of MAHs into separate paragraphs.

1) Pragmatic $U X$ : Pragmatic UX refers to everything related to achieving behavioral goals, it refers to effectiveness, efficiency, subjective workload, controllability, predictability and more [12] [22]. So far, studies are showing diverging results concerning the usability and pragmatic value of MAH feedback. In a driving simulator, MAH feedback during gesture-based interaction with an infotainment system led to a higher accuracy in slider bar tasks, compared to when MAH feedback was absent [3]. Also in another study involving an automotive context, the addition of MAH feedback to a virtual touchscreen led to significant benefits in primary task performance (e.g. the number of off-road glances) [23]. Apart from 
the automotive context however, results are less supportive of an added value of MAHs. For example, when adding MAH feedback to selecting gestures in VR, no performance benefits were observed [24]. When considering subjective workload, Sand et al. [24] observed a significant decrease in temporal demand (subscale of workload), but not workload in general, when MAH feedback was added. In a recent study, involving a gesture-based interface, there was no significant pragmatic advantage of MAH feedback on top of visual feedback, but MAH feedback did lead to lower workload when compared to a condition without any feedback at all [5]. When considering a study investigating the added value of haptic feedback when used to enhance audio-visual content, no significant difference in pragmatic UX was observed when adding MAH sensations to the videos [15].

2) Hedonic UX: Hedonic UX involves enjoyment, identification (communication of identity to relevant others, relatedness), stimulation, evocation (provoking memories, symbolizing) and more [25][22][26]. Compared to pragmatic UX, less research has been done on the added value of MAH feedback concerning the hedonic experience. In one study, adding MAH feedback while playing a virtual piano led to significant increases in enjoyment [27], and in another study MAH feedback significantly increased the attractiveness of a gesture-based interface [5]. However, after statistically controlling for perceived novelty in the latter study, attractiveness was no longer significantly elevated. Furthermore, Maggioni et al. [15] did not observe a significant difference in hedonic UX when comparing audio-visual content with versus without MAH enhancement.

3) Emotional Reactions: For users' emotional reactions, we refer to the arousal and valence of the emotional reaction, assessed with the self-assessment manikin [28], its updated version: the affective slider [29], the affect grid [30], and skin conductance response (SCR). MAH feedback has been found to significantly impact the arousal of users' emotional reactions. Ablart et al. [11] investigated the impact of adding MAH sensations while watching 1-minute movies at two points in time, with an interval of two weeks. They observed no significant impact of MAH sensations on valence of the emotional reaction while watching a movie, but self-reported arousal significantly increased when MAH sensations were present. Interestingly, this significant increase in self-reported arousal was evident both during the first and second session, so it did not wear off over time. Arousal implicitly assessed as skin conductance response was not elevated when MAH feedback was present, but was overall (whether or not MAH feedback was present) significantly higher in the first, compared to the second session. In another study considering haptically enhanced audio-visual content (6-second movies), no differences in arousal or valence of the emotional reactions were found when MAH feedback was present [15]. When considering MAH sensations while interacting with digital kiosks, again no significant difference was found regarding the arousal and valence of users' emotional responses [9]. In the context of a gesture-based interface, participants reported a significantly higher valence of the emotional reaction (pleasure) when MAH feedback was added on top of visual feedback, but this effect disappeared after statistically controlling for perceived novelty [5].

With the current study, we add to the existing literature by assessing a variety of pragmatic and hedonic UX qualities, as well as the arousal and valence of users' emotional reaction at two points in time: during initial experience with MAHs and after repeated use. Except for Ablart et al. [11], where only the emotional reactions were assessed, this study is the first to report how the UX of MAH feedback changes over time.

\section{B. The Novelty Effect}

Although it is an important challenge when investigating the UX of a new technology, only little research actually addresses the novelty effect [10]. When considering the UX of MAH feedback, only two studies took the novelty effect into account. In one study, self-reported arousal remained elevated after repeatedly experiencing MAH feedback while watching a movie [11]. They interpreted a general drop in skin conductance response (SCR) as a lowering of the novelty effect. In a second study [5], perceived novelty was assessed using the User Experience Questionnaire (UEQ) [22]. When statistically controlling for perceived novelty the added value of $\mathrm{MAH}$ feedback with regard to attractiveness and pleasure disappeared.

As mentioned earlier, a general consensus on its definition or operationalization does not exist within HCI, but based on the description provided by Koch et al. (see Introduction) [10], we would describe the novelty effect in the context of adding MAH feedback to a system as: an increase in the perceived pragmatic and/or hedonic value, as well as an increase in the arousal and/or valence of the users' emotional response, on account of its newness. When novelty eventually fades, the increased UX qualities and arousal and valence of the emotional responses disappear. The novelty effect highlights the importance of considering the temporality of the user experience, i.e. the user experience changing over time [31]. Indeed, UX should not be considered a static entity, but instead it has a dynamic nature [32]. Studies taking temporality into account inevitably need to invest in a longitudinal approach, which is very time-consuming and challenging (e.g. to avoid drop-out) and therefore rather scarce. However, such studies yield valuable results. For example, it has been found that the UX qualities driving prolonged versus initial use of the iPhone were very different, with stimulation (excitement) and learnability (frustration) being mostly salient during initial use, whereas usefulness became more salient during a second phase. During the third and last phase, the emphasis shifted from usefulness and long-term usability to personalization and self-expression through the device [31]. Similarly, the UTAUT assigns an important role to the amount of experience one has with the novel device (experience during first encounter, after 1 month and after 3 months). Experience has been found to be a moderating variable on e.g. the relationship between effort expectancy and intention to use a new technology. The less experienced with a new technology, the larger the impact of effort expectancy on intention to use [17]. Also when considering emotional responses, it is important to take into account their 
temporality. Kujala and Miron-Shatz [33] investigated how people's emotional responses changed over a period of five months with regard to using a mobile phone. They observed that during initial use, participants overestimated their positive emotions and focused more on positive than negative emotions [33].

Apart from these global tendencies in UX and emotional responses over time, there is also evidence for individual differences in the change patterns. Harbich and Hassenzahl [32] conducted an elaborate longitudinal field study focusing on the user experience of interactive work-related products (e.g. Microsoft Office Visio 2003), with 12 points of UX measurement captured over a period of 13 weeks. They observed considerable variance between participants regarding the way different UX qualities changed over time. For example, the variable 'execute', reflecting the product's ability to support the achievement of goals, showed an increasing variance between individuals over time, with the absence of a consistent, global pattern of change. This meant that some participants struggled less, but others struggled more with the use of the product over time. When considering engagement, reflecting the product's ability to support motivation and persistence at a task, there was again considerable variance between individuals over time but also a consistent, global decrease over time [32]. In particular, the decline in engagement was more pronounced for more playful participants as they lost their interest in the product faster than less playful participants. Apart from large inter-individual differences in the rate and direction of change of UX qualities regarding work-related products, Harbich and Hassenzahl also observed an overall trend of the UX getting worse over time [32].

Evidently, the novelty effect is closely related to temporality, but after how many weeks can one conclude that the novelty effect wore off? How can we assess the novelty effect? As mentioned earlier, there is no consensus about how the presence of the novelty effect should be identified. As stated by Koch et al. [10], the novelty effect has a complex nature and is mostly present on two occasions: 1) at the moment the new device or system gets implemented and 2) every time changes are made to an existing device or system. They described different approaches that could be used to assess the product's novelty: for example, novelty could refer to system features that the users perceived as surprising or unfamiliar [34], or novelty could be conceptualized as a user experience dimension, such as perceived novelty from the UEQ [22] [5]. Furthermore, novelty could be assessed as the transition from an initial spike in usage to more stabilized usage levels [10][35]. Often however, instead of identifying the presence of a novelty effect through a formal assessment, it is assumed to fade over the course of a couple of weeks or months. In Harbich and Hassenzahl's study [32], the presence of the novelty effect was not formally assessed, but they assumed that it wore off after 13 weeks. In Karapanos et al. [31], the temporality of the user experience of the new iPhone was assessed over a course of five weeks. Research on the user experience of public displays recommends a period of four weeks in order for the novelty effect to fade [36]. Koch et al. [10] reported that in one of their studies concerning the UX of public displays, it took about ten weeks for the novelty effect to fade, whereas in a second study, it only took about one week. They concluded that the duration of behavioral changes due to the novelty effect depends on a variety of variables and the application context.

The methodological contribution of this study lies in exploring whether perceived novelty from the UEQ can be used to identify the presence of the novelty effect [22] [5]. With this goal, we address the challenge expressed by Koch et al. [10] that more research is needed to investigate a standard to identify and assess the novelty effect's presence.

\section{The Current Study}

The aim of the current study was twofold. First, we investigated how MAH feedback, when added to a gesture-based interface, would impact users' emotional responses (valence and arousal) and the pragmatic and hedonic UX. We tested whether this impact of MAH feedback would be different during initial experience versus after repeated experience. In short, our first research question was: what is the added value of MAH feedback and does it disappear after repeated use? In particular, we expected an added value of MAH feedback concerning enjoyment and engagement, but expected these effects to disappear over time due to a novelty effect. Similarly, we expected users to perceive the home automation system as significantly more hedonic when MAH feedback was added, but again only during initial use (novelty effect). Indeed, earlier research has shown that MAH feedback leads to a higher attractiveness of a gesture-based interface [5] and to greater engagement with digital kiosks [9]. However after statistically controlling for perceived novelty, the increased attractiveness disappeared [5]. Moreover, in Harbich and Hassenzahl's study [32], engagement significantly dropped over time. Concerning pragmatic UX (usefulness and ease of use), as it was assessed outside of an automotive context in the current study, we did not expect an added value of MAH feedback [15] [5]. When considering users' emotional reactions, we did expect significantly increased valence when MAH feedback was present, but due to a novelty effect [5]. When considering arousal, we again expected a significant elevation when MAH feedback was present, but no novelty effect because earlier research has shown that self-reported arousal remained elevated over time [11]. See Table 1 for a schematic overview of these hypotheses related to the first research question. Our second research question was: can perceived novelty be used as a way to identify the presence of a novelty effect? In order to reflect the presence of a novelty effect, we expected perceived novelty to be significantly increased when MAH feedback was present during the first session, but not during the last session because after repeated use, the novelty effect should wear off.

To address these research questions, we conducted a longitudinal lab study over the course of five weeks. We chose five weeks based on earlier research, where a decrease of the novelty effect was already observed after four weeks [36], after two weeks [11], or even after one week [10]. As MAH feedback is not yet implemented in everyday devices, the study had to be conducted in-lab. To ensure elaborate 
interaction with the MAH sensations, all participants attended eight sessions of interacting with the gesture-based device. The gesture-based device consisted of a fictitious home automation system, where the MAH feedback could be turned on or off so we could assess the user experience when MAH feedback was present versus absent. In line with two application categories of MAH feedback, as described by Van den Bogaert et al. [37], we applied MAH feedback to indicate a changing status, and to provide information about the parameters they could manipulate in the home automation system (see Methods, Materials, Experimental Task).

TABLE I: Schematic Overview of the Hypotheses linked to Research Question 1

\begin{tabular}{lll}
\hline DV & impact MAH & novelty effect \\
\hline Enjoyment & yes & yes \\
Engagement & yes & yes \\
User conception & yes & yes \\
Usefulness & no & no \\
Ease of use & no & no \\
Continued Use & exploratory & exploratory \\
Valence & yes & yes \\
Arousal & yes & no
\end{tabular}

Note. DV = dependent variable. Impact MAH refers to whether or not we expected an added value of MAH feedback, novelty effect refers to whether or not we expected the added value to be due to a novelty effect. Exploratory refers to the fact we included the variable continued use for exploratory reasons.

\section{METHODS}

\section{A. Participants}

Since participants needed to visit our lab repeatedly during a five-week period, we recruited students following courses at the same campus where the study took place. Moreover, as we focused on MAH feedback in a home automation system, which is relevant for a broad target audience, and previous research has shown that the ability to clearly perceive $\mathrm{MAH}$ sensations strongly decreased with increasing age [38], we considered a young sample, in this case students, recommended. A total of 31 students were selected, based on their availability during that five week period. Upon being accepted as a participant, everyone had to confirm their presence during eight sessions through an anonymous Doodle. One participant dropped out during the course of the study, a second participant gave an incorrect response on the exclusion criterion item (see Procedure), and a third participant had to be excluded because of insufficient knowledge of the language in which the study was conducted. Of the 28 remaining participants, the mean age was 20.7. Six participants were male, 22 were female. Exclusion criteria for participation were: previous experience with MAH technology, and having touch deficits in the upper limbs. This study was approved by the local social and societal ethics committee: G- 2019101780.

\section{B. Materials}

1) Experimental Task: For the experimental task, participants had to interact with a gesture-based (fictitious) home automation system, presented on a laptop monitor. Mid-air haptic sensations, based on ultrasound waves, were added to the home automation system, using a Stratos Development kit (UltraLeap), see Figure 1 (left) for the experimental set-up. With this set-up we tried to mimic a home automation system fixed to the wall. The MAH device was placed at a height of about $80 \mathrm{~cm}$, with the height of the laptop at about 130 $\mathrm{cm}$. The lengths of the different slider bars ranged from about $12 \mathrm{~cm}$ to $19 \mathrm{~cm}$. The time it took to adjust each slider bar ranged from 2 to 10 seconds, depending on the task (selecting a precise temperature took longer than changing the lights).
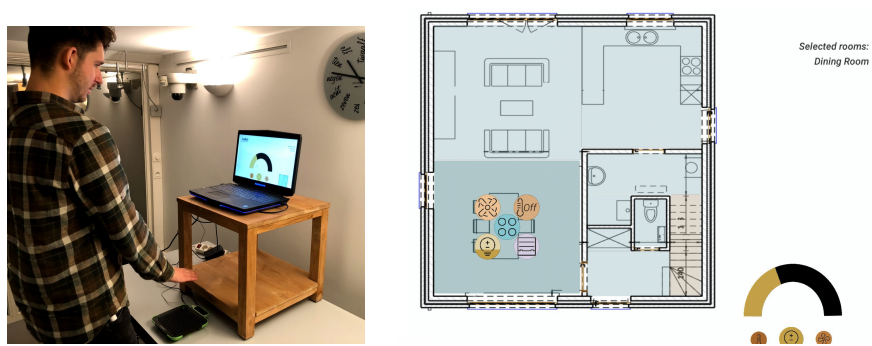

Fig. 1: Left: Experimental set-up. A participant is changing the lights by manipulating a slider bar using hand gestures. Right: The floor plan of the home automation system's interface, with a selected room and parameter: the dining room and the slider bar to adjust the lights. After confirming a selected room, the slider bar appears enlarged in the middle of the screen, see left figure.

We could turn the mid-air haptic sensations on or off, which allowed us to easily switch between the two conditions in our study: the system with and without MAHs. The interface showed a floor plan of a house (see Figure 1 - right), with the possibility of switching between the ground floor and the first floor. Rooms on both the ground and first floor could be selected to adjust four different parameters: the lights, the curtains, the air conditioning and temperature. Input to this device was given through hand gestures. The hand gestures were chosen based on their simplicity and suitability in terms of facilitating the perception of MAH sensations. As MAH sensations are best perceived on the palm of the hand or finger tips (and not the back or sides of the hand), almost all gestures involved having the palm of the hand directed downwards (in the direction of the ultrasound waves). For example, the lights could be dimmed by sliding with the index finger over a slider bar, or the air conditioning could be adjusted by hovering with the palm of the hand over two buttons: one to increase the fan speed, and the other to lower the fan speed. As previous work has shown that shape identification is not easy [38], at least when presented without a dynamic tactile pointer [39], we did not create different MAH shapes to go along the gestures. Instead, we relied on MAH patterns that all had the same circle-like shape. Depending on the gesture it was combined with, it could have a variable or invariable intensity, and it could be projected on the palm of the hand or the index finger tip. MAH feedback was provided for all gestures involved in changing the parameter settings and when selecting rooms, but not when switching between the 
ground and the first floor because the swipe gesture led to a vertical position of the hand above the MAH device, inhibiting the perception of MAHs. MAH sensations used to provide information (application category 3) [37] involved a sensation on the palm of the hand when hovering above selected rooms, informing the user about which rooms were selected and which were not (no sensation). MAH sensations used to indicate a changing status (application category 5)[37] were provided while manipulating the household parameters. Each time the fan speed was increased or decreased by one setting, a discrete sensation was felt on the palm of the hand. When operating the temperature or curtains, a pulsating sensation was felt. When increasing the brightness of the lights, the MAH sensation on the finger tips increased in intensity, whereas when dimming the lights, it decreased in intensity. To standardize the way participants would interact with the system, we wrote a number of different assignments (or experimental tasks), related to each of the four parameters, on small cards. The cards were divided into four decks, one for each parameter. This way, participants could draw different assignments for each of the four parameters. An example of an assignment would be "You have visitors tonight. Set the temperature of the living and dining room to a comfortable degree $\left(21^{\circ} \mathrm{C}\right)$ ".

2) Questionnaires: The questionnaires were administered both in the introductory and the closing session, after each of the two conditions (with/without MAHs). Thus, we obtained four questionnaires from each participant. This way, we could compare participants' experience with the device with MAHs versus without MAHs, in both the introductory and closing session. Each questionnaire started with an exclusion criterion item, where participants had to indicate whether they just performed the condition with or without MAHs. Participants choosing the wrong option from the drop-down menu would be excluded from analysis. To assess the pragmatic UX, we relied on perceived usefulness and perceived ease of use, as defined by the TAM [16] [19], translated to respectively five and four items presented on a 7-point rating scale. The hedonic UX was operationalized as enjoyment and assessed using four items on a 7-point rating scale [18]. Continued use was assessed using three items on a 7-point rating scale following Kose et al. [18] and Turel et al. [40], and user conception, or the degree to which the system was perceived as hedonic (versus utilitarian), was assessed as a 7-point semantic differential scale consisting of 2 items [18]. The valence and arousal of users' emotional reactions were quantified using the Affective Slider, both rated on a scale from 0-100 [29]. Following Limerick et al. [9], we also incorporated the User Engagement Scale - short form (UES-SF; [21] [41]) as a way to assess user engagement. Finally, perceived novelty was assessed using a subscale of the User Experience Questionnaire - short (UEQ-S), consisting of four items on a 7-point Likert rating scale [22]. All outcome variables, except for valence and arousal, were computed by taking the average score of their respective items. E.g. the outcome variable usefulness was computed as the average score of its five respective items.

3) Interview Questions: In addition to the questionnaires, we also included some open-ended questions which were audio recorded and transcribed for thematic analysis. The reason these questions were included was to allow participants to elaborate further on their responses given in the questionnaires, with the aim of obtaining richer information regarding their experience with the device with/without MAHs. The interview questions were therefore based on aspects of the UX as assessed by the questionnaires. We asked about "efficiency" (i.e., "did you find the home automation interface more or less efficient with or without the MAHs? And why?"), and in the same fashion "ease of use", "enjoyment" and about users' intention to continue using the system. We also asked about their overall preference for the home automation system with or without MAHs. All these questions were asked in both the introductory and closing session.

\section{Procedure}

This study consisted of eight sessions: two long sessions of about 40 - 45 minutes each: the first (introductory) session and the last (closing) session, and six shorter sessions of about 15 minutes each. In each of the eight sessions, participants interacted with the home automation system (and performed the experimental tasks) during about 15 minutes. This was the sole activity during the shorter sessions. During the longer introductory and closing sessions, participants also completed questionnaires and responded to interview questions. Interacting with the home automation system, the recurrent activity during each session, always followed the same course. Participants were assigned in counterbalanced order to both conditions (with \& without MAHs) in each session (withinparticipant design). In each condition, they completed four tasks by randomly drawing one card from each of the above mentioned card decks, with each deck including tasks for one specific household parameter. This meant they completed four tasks, each related to a different household parameter, in each condition. Thus, after completing both conditions in each session, they completed eight different tasks (four tasks with and four tasks without MAH feedback). In addition to the eight experimental tasks, the introductory and closing session involved some extra elements.

After signing the informed consent in the introductory session, participants experienced their first MAH sensations, which were templates from Ultraleap's sensation editor. After this short familiarization phase, participants were introduced to the home automation system without MAHs. We explained them how to navigate through the interface, and participants could train the necessary gestures. When they understood how the interface worked, the actual experimental tasks commenced. After finishing the four tasks in the first condition, participants filled out the questionnaire asking about their experience, starting with the exclusion criterion item, and the rest of the questions presented in randomized order (see Questionnaires). After finishing the questionnaire, participants completed the second condition, and filled out the same questionnaire. Thus, we obtained two questionnaire of each participant: one about their experience with and one about their experience without MAH feedback. Finally, this session ended with some open-ended questions: first we asked about their global preference (with/without MAHs), and next about 
more specific aspects of their experience (e.g. usefulness, ease of use) (see Interview Questions). Participants received a $€ 10$ bol.com voucher as incentive. The closing session followed the same procedure as the introductory session, but without the familiarization phase and the introduction to the home automation system. Participants who attended all eight sessions received a $€ 40$ bol.com voucher during this last session.

\section{RESUlts}

\section{A. Questionnaire Data}

The statistical analyses were performed on complete questionnaire data of 28 participants across both sessions. To answer research question 1 , we first tested how the home automation system was experienced with versus without MAHs at both sessions, informing us about the potential added value of MAHs (main effect of condition). Second, we tested whether the UX and emotional reactions to the home automation system in general would be different between initial encounter and after repeated use (main effect of session), and third, we investigated whether the added value of MAH feedback would disappear during the last session (interaction effect of condition and session). This interaction effect would show which aspects of the added value of MAH feedback were vulnerable to a novelty effect. We analyzed the data using repeated measures ANOVA (RM ANOVA; ez package in R [42]) with condition (with/without MAHs) and session (session 1/session 8) as independent variables, and the different UX components (e.g. enjoyment, usefulness, ease of use), and emotional reactions (valence and arousal) as dependent variables ${ }^{1}$. For each dependent variable, a separate RM ANOVA was performed. To address research question 2: whether perceived novelty of the UEQ could reflect the presence of a novelty effect, we first tested whether the device with MAH feedback was perceived as more novel than the device without MAH feedback (main effect of condition). Second, we tested whether perceived novelty decreased over time (main effect of session), and third, whether the significantly elevated perceived novelty of MAH feedback at session 1 would disappear at session 8 (interaction effect of condition and session). A significant interaction effect between condition and session would support perceived novelty as a way to identify the presence of a novelty effect. Again, the data were analyzed using RM ANOVA, with condition and session as independent variables, and perceived novelty as dependent variable.

\footnotetext{
${ }^{1}$ Concerning the RM ANOVA assumptions, given the $2 \times 2$ design, the sphericity assumption was automatically met. Furthermore, ANOVA has been found to be fairly robust with regard to violation of normality and homogeneity of variances in the case of equal group sizes and at least 20 degrees of freedom [43][44][45]. However, we did verify whether our results were robust by conducting a non-parametric variant of RM ANOVA: permutation tests using the ezPerm function (ez; [42]). The results obtained with the parametric and non-parametric variant were very comparable, with the same main and interaction effects being significant/non-significant. In other words, the results of the analyses were robust and did not meaningfully change depending on the type of analysis used. Note however that the ezPerm function is work in progress and only the main effects may be trusted.
}

1) Hedonic $U X$ and Engagement: The RM ANOVA with enjoyment (hedonic UX) as dependent variable showed no significant interaction effect between condition and session, $F(1,27)=1.18, p=.29$. There was a significant main effect of condition however, $F(1,27)=8.97, p<.01, \eta_{G}^{2}=.02$, which means that we observed an added value of MAHs in terms of increased enjoyment while interacting with the home automation system across both sessions. There was also a significant main effect of session, $F(1,27)=15.10, p<$ $.001, \eta_{G}^{2}=.08$, meaning that participants rated the experience with the home automation system in general as more enjoyable during session 1 compared to session 8 (see Figure 2). User conception, or the degree to which the system was perceived as hedonic (versus utilitarian) showed no interaction effect between condition and session, $F(1,27)=0.05, p=.83$, and no main effect of session, $F(1,27)=0.25, p=.62$. There was a main effect of condition however, $F(1,27)=$ $9.32, p<.01, \eta_{G}^{2}=.03$. Participants perceived the home automation system with MAHs as more hedonic than the device without MAHs, also after repeated use (see Figure 2). The results regarding engagement were similar: no interaction effect between condition and session, $F(1,27)=0.24, p=$ .63 and no main effect of session, $F(1,27)=0.54, p=$ .47 , but there was a significant main effect of condition, $F(1,27)=7.17, p=.01, \eta_{G}^{2}=.04$. Participants reported more engagement with the home automation system when MAHs were present versus absent, both after initial use, and after repeated use. This means there was an added value of MAHs in terms of engagement (see Figure 2).

2) Pragmatic UX and Continued Use: Considering pragmatic UX: usefulness and ease of use as dependent variables, we observed again no interactions effects, $F(1,27)=$ $0.16, p=.69$ for usefulness, and $F(1,27)=0.28, p=.60$ for ease of use. For usefulness, there was no main effect of session, $F(1,27)=0.10, p=.75$, meaning that there was no change in the perceived usefulness of the home automation system between session 1 and session 8. However, in general, participants rated the home automation system without MAHs as more useful than with MAHs, as evident in the main effect of condition, $F(1,27)=7.17, p=.01, \eta_{G}^{2}=.005$ (see Figure $2)$. For ease of use there was no significant main effect of condition, $F(1,27)=0.04, p=.85$, meaning that MAHs did not impact the ease of use, but there was a main effect of session, $F(1,27)=7.32, p=.01, \eta_{G}^{2}=.06$, with a higher ease of use during session 8 compared to session 1 (see Figure 2). In short, MAHs did not show an added value in terms of usefulness or ease of use based on these results. Continued use showed no main effects nor interaction effects, with $F(1,27)=0.00, p=.95$ for condition, $F(1,27)=$ $0.09, p=.77$ for session, and $F(1,27)=1.71, p=.20$ for the interaction between both. This means that participants indicated that MAHs did not have any impact on whether or not they thought they would continue using the home automation system. Also the amount of experience with the device (initial versus after repeated use) did not impact their intention to continue using the device (see Figure 2).

3) Emotional Reactions: When considering participants' emotional reactions in terms of valence and arousal, some 

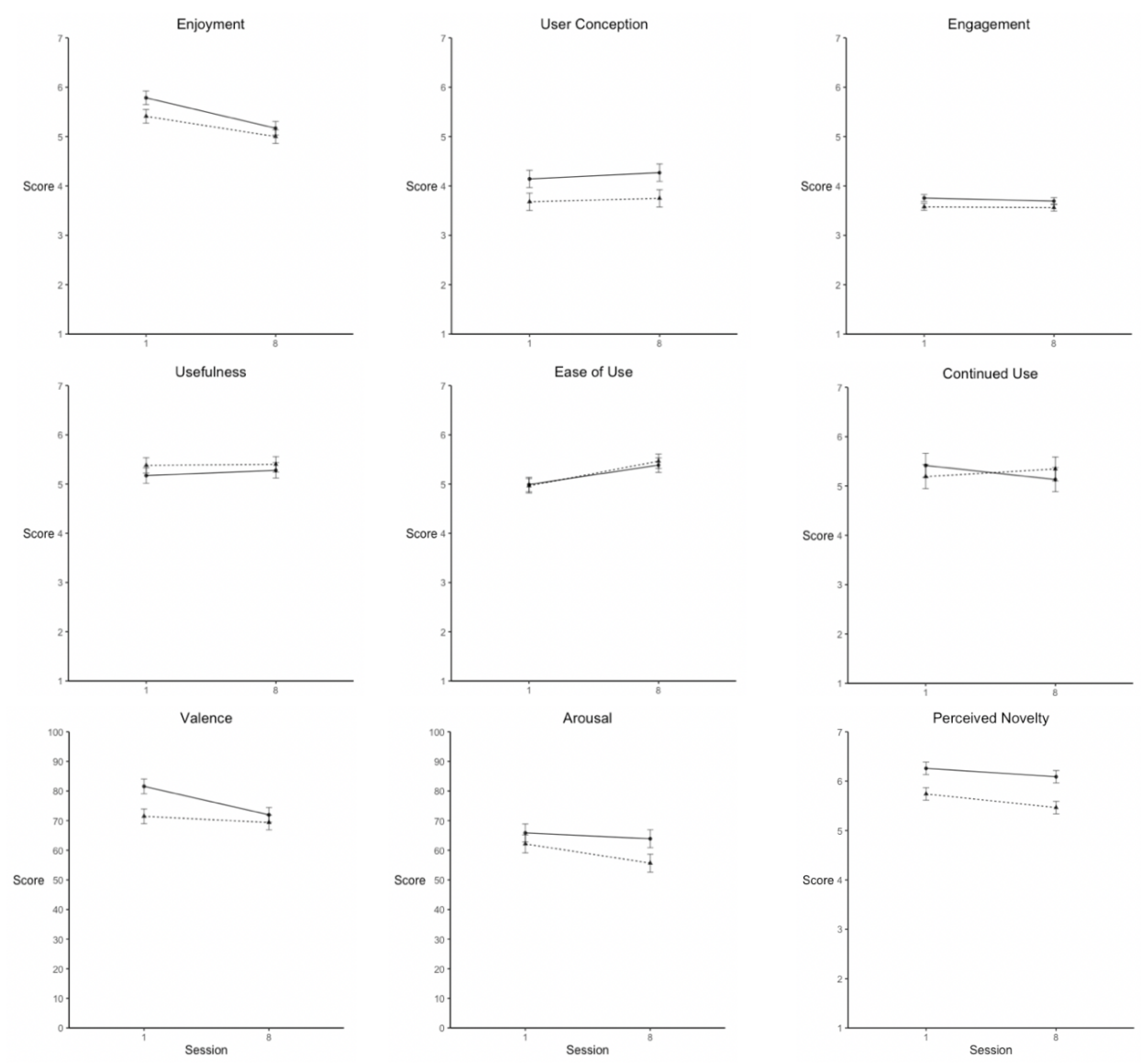

Fig. 2: The impact of condition (with versus without MAHs) and session on the different dependent variables (computed as the average scores of the respective items, see Questionnaires). The error bars are based on Fisher's Least Significant Difference [42].

interesting results were observed. Valence as dependent variable revealed a significant interaction effect between condition and session, $F(1,27)=4.82, p=.04, \eta_{G}^{2}=.02$, as well as two main effects, with $F(1,27)=8.11, p<.01, \eta_{G}^{2}=.05$ for condition, and $F(1,27)=7.61, p=.01, \eta_{G}^{2}=.04$ for session (see Figure 2). However, given the significant interaction effect, we did not interpret the main effects. Posthoc paired t-tests showed that condition had a significant impact on valence ${ }^{2}$, but only during session $1, t(27)=$ $3.85, p<.001$, Cohen's $\mathrm{d}=0.73$. During session 8 , this was no longer statistically significant, $t(27)=0.87, p=.39$. This means that MAHs showed an added value in terms of the valence (pleasure) experienced during the interaction, but only during initial use. The added value of valence (pleasure) disappeared after repeated use. For arousal, the results were quite different: no interaction effect between condition and session, $F(1,27)=1.18, p=.29$, no main effect of session, $F(1,27)=2.44, p=.13$, only a significant main effect of condition, $F(1,27)=5.40, p=.03, \eta_{G}^{2}=.02$. Across both sessions, participants experienced more arousal when MAHs were present versus absent, and thus a general added value of

\footnotetext{
${ }^{2}$ Bonferonni correction: $\alpha=.05 / 2=.025$
}

MAHs in terms of arousal (see Figure 2).

4) Perceived Novelty: Interestingly, we observed no interaction effect between condition and session when perceived novelty was used as dependent variable, $F(1,27)=0.38, p=.54$, and surprisingly, there was no main effect of session either, $F(1,27)=3.33, p=.08$. There was a significant main effect of condition however, $F(1,27)=21.21, p<.001, \eta_{G}^{2}=.13$. This means that the home automation system with MAHs was perceived as more novel compared to the system without MAHs, both during initial use, and after repeated use. Furthermore, perceived novelty did not fade over the course of five weeks (see Figure 2).

\section{B. Interview Data}

The interview data for a large part supported the findings described above, but at the same time revealed meaningful nuance and ambiguity underpinning the questionnaire data. Heightened enjoyment as shown by the statistical analyses, for example, was indeed something that was touched upon during the interview by a majority of participants, albeit not necessarily in those words. Eight participants described the MAH sensations as "fun", and 14 noted that the interface was 
"more pleasant" with the MAH sensations. Another interesting description was used by seven participants, they referred to a "heightened level of involvement with the interface", which supported the quantitative findings regarding "engagement". Conversely, there were participants who explicitly stated that they did not enjoy the MAH sensations. This showed that the general findings of MAH feedback increasing enjoyment while interacting, might occlude interpersonal differences. Two participants stated that the MAH feedback induced frustration, and four others literally described the interface as less pleasant with MAH feedback. Without it necessarily having a negative impact on enjoyment, more than half of the participants $(n=15)$ also mentioned that, at least at first, the MAH sensations were a bit startling and thereby distracting. With regards to the pragmatic UX components, the interviews confirmed the questionnaire data, but revealed some more details about them. Two main aspects of pragmatic UX were mentioned in particular: functionality (or usefulness) and ease of use. The questionnaire data showed that usefulness was perceived as higher without MAH feedback. In line with this, nine participants made it explicit that that even though they preferred the home automation system with MAH feedback for hedonic reasons, they simultaneously found that there was no pragmatic benefit of the MAHs or, even more strongly, that the MAH feedback negatively impacted the pragmatic facets of the UX. Others $(n=3)$ however declared the exact opposite, again clearly showing interpersonal differences. They described the MAH feedback as "making the interface more clear", "heightening the sense of agency" or "lowering the cognitive load", while simultaneously being "unpleasant" or "distracting". In addition to highlighting interpersonal differences, this also shows that, at least in the case of MAH feedback, the hedonic and pragmatic UX qualities were not necessarily in line with each other. Concerning the temporal component, the interviews clarified how and why MAH feedback was preferred either in the beginning session or only later on. Six participants mentioned how the MAH feedback had a "pedagogical" functionality by helping them to get acquainted with the home automation system and the gestures used to control it. Others $(n=7)$ experienced the MAH feedback as distracting at first, but grew fond of it once they got used to it. Some of them mentioned a "learning curve" that they had to go through.

\section{Discussion}

An overview of the results regarding the hypotheses related to research question 1 are provided in Table 2. There was an added value of MAH feedback in terms of enjoyment (main effect of condition), which did not disappear over time, indicating the absence of a novelty effect (no interaction effect between condition and session). Overall though (whether or not MAH feedback was present), participants reported significantly less enjoyment at session 8 (main effect of session). Moreover, participants perceived the device with MAH feedback as significantly more hedonic (see DV user conception) than without MAH feedback (main effect of condition), which did not disappear over time (no interaction and no main effect of session). These results were only partly in line with our hypothesis as we predicted that the added value of MAH feedback in terms of hedonic UX qualities would be susceptible to a novelty effect. This was based on an earlier study showing the disappearance of increased attractiveness of the interface involving MAH feedback after statistically controlling for perceived novelty [5].

TABLE II: Schematic overview of the results regarding the hypotheses linked to research question 1

\begin{tabular}{llll}
\hline DV & impact MAH & novelty effect & impact time \\
\hline Enjoyment & higher & no & lower \\
User conception & higher & no & no \\
Engagement & higher & no & no \\
Usefulness & lower & no & no \\
Ease of use & no & no & higher \\
Continued Use & no & no & no \\
Valence & higher & yes & lower \\
Arousal & higher & no & no \\
\hline
\end{tabular}

Note. $\mathrm{DV}=$ dependent variable, impact MAH refers to a main effect of condition (with/without MAH), with higher indicating that MAH feedback led to a higher score on that outcome variable and lower indicating that MAH feedback led to a lower score on that outcome variable. Novelty effect refers to whether or not the impact of MAH feedback was due to a novelty effect (reflected in an interaction effect between condition and session). Impact time refers to a main effect of session (session 1/session 8), with lower indicating that the outcome variable had a lower score over time, and higher, that it had a higher score over time.

Similarly for engagement, based on earlier work, we expected an added value of MAH feedback [9] that would disappear over time [32], but we only observed a main effect of condition (and no main effect of session or interaction effect). This means that MAH feedback significantly increased the engagement with the home automation system, both during initial use and after repeated use, replicating and extending the findings of Limerick et al. [9], where engagement with digital kiosks was elevated when MAH sensations were present. However, they only assessed engagement during an initial encounter. So it appears that MAH feedback leads to greater engagement both in the context of interacting with a home automation system, and when interacting with digital kiosks, without being susceptible to a novelty effect. This appears to contradict the results of Harbich and Hassenzahl [32]. However, in their study engagement was conceptualized as a work-related product's ability to support motivation and persistence at a task, whereas in ours and Limerick's study [9], it was assessed using the UES-SF [41], consisting of items assessing aesthetic appeal, focused attention, perceived usability, and reward [20][21]. Clearly, those two engagement conceptualizations tap into different qualities and were assessed in different contexts, which could explain the diverging results.

The results regarding pragmatic UX were quite in line with our predictions. For usefulness though, there was a main effect of condition which we did not predict. However, this main effect did not reflect an added value of MAHs, rather the other way around. Indeed, participants perceived the device without MAHs as more useful than with, and this perception 
of usefulness did not change over time (no main effect of session nor interaction effect). There was no added value of MAHs regarding ease of use (no main effect of condition and no interaction effect), but generally, whether or not MAH feedback was present, the ease of use increased over time (main effect of session), which probably reflected a learning effect. Continued use, included for exploratory reasons, revealed that MAH feedback had no impact on participants' tendency to keep using the device, both during initial encounter and after repeated use (no main or interaction effects).

In line with our hypothesis and earlier research [5] [33], we observed an increase in the valence of the emotional response (higher pleasure) when MAH feedback was present, but only during first encounter. After repeated use, the added value of MAH feedback in terms of valence disappeared, indicating the presence of a novelty effect (interaction effect between condition and session). When comparing the results of valence with enjoyment (hedonic UX quality), one can wonder why a discrepancy was observed. Valence was impacted by a novelty effect, but enjoyment was not. Indeed both variables appear to assess similar aspects of the UX. However, enjoyment is considered a hedonic UX quality, whereas valence of the emotional response is about users' emotional reaction. Enjoyment consists of items such as "I find using the device interesting" and "I find using the device exciting", where participants have to indicate the extent to which they agreed with the statements [18]. Contrarily, valence of the emotional response is typically assessed using a pictorial scale, such as with the Self-Assessment Manikin [28] or the Affective Slider [29]. Thus, although both variables appear to resemble each other at first sight, they do tap into different qualities. Again in line with our hypothesis and earlier research [11], adding MAH feedback to the home automation system led to significantly increased arousal of the emotional response (main effect of condition), which did not disappear after repeated use (no main effect of session or interaction effect).

Concerning research question 2, we explored whether perceived novelty (UEQ) could be used as a way to identify the presence of a novelty effect. Our results showed that perceived novelty was significantly elevated when MAH feedback was present, not only during the initial encounter but also after repeated use (only main effect of condition, no interaction effect). Moreover, perceived novelty did not significantly decrease over the course of five weeks (no main effect of session). These results were not expected based on the literature, where the novelty effect has been found to fade after a period of four weeks, two weeks or even one week [36] [10] [11], meaning that after five weeks we should have observed a significant decrease in perceived novelty if it would have reflected the presence of the novelty effect. One could wonder whether five weeks were actually enough for the novelty effect to fade. However, five weeks were enough in our study to observe how the novelty effect considering valence of the emotional response wore off. Therefore, we would argue that perceived novelty (UEQ) can not be used to identify the presence of a novelty effect in a comprehensive way. We would speculate that although perceived novelty (UEQ) might be good at capturing the consciously perceived novelty and novel features of a system or device, the novelty effect might relate to a less conscious experience. Future research should focus on developing an assessment tool with the specific aim of identifying the presence of a novelty effect. This could be done by developing a questionnaire or by investigating implicit tools, such as skin conductance response (SCR) [11]. However, in Ablart et al. [11] SCR was not significantly elevated when MAH sensations were present (versus absent), not even during the initial encounter. SCR did drop in general though (irrespective of the presence or absence of MAH sensations) from session 1 to session 2 . We believe that a comprehensive operationalization of the novelty effect concerning MAH feedback should address two phases, similar to the description by Koch et al. [10]. First, during initial experience of MAH sensations, there should be a significant impact on the concept used to operationalize novelty (perceived novelty in the current study). Second, after repeated use, this effect should disappear, reflecting the habituation of MAH sensations.

The analysis of the interview data suggested that even though the questionnaire data revealed interesting general trends, they did not necessarily tell the whole story. In our case, a set of open-ended questions showed that "preference or no preference" for MAH feedback was often no clearcut matter. Moreover, for twelve participants, hedonic and pragmatic UX were in clear contrast with each other. Nine of them expressed a preference for MAH feedback for hedonic reasons, but at the same time mentioned that there was no pragmatic benefit (or even more strongly: a negative impact of MAH feedback on the pragmatic UX). Just as well, the opposite was mentioned by the other three participants who said that they found the interface more clear and practical with the MAH feedback, even though these tactile sensations were not perceived as hedonically enjoyable. This showed that hedonic and pragmatic features of the UX with regard to MAH feedback are not necessarily intertwined. Individual differences regarding the UX of work-related products were observed by Harbich and Hassenzahl [32] as well. They showed that over time, engagement with the product generally dropped, but interestingly, this was more strongly the case for more playful compared to less playful users. Moreover, they observed that some users struggled more when using the product, whereas others struggled less over time.

\section{A. Implications for Design}

Based on the individual differences we observed in the interview data, it appeared that a preference for having MAH feedback implemented or not in gesture-based systems differed between individuals, with some liking MAH feedback for hedonic reasons and disliking it for pragmatic reasons or the other way around. Thus, similar to current commercial devices having the option to turn vibrotactile feedback on or off, such an option might be worth considering in the case of interfaces with a MAH component was well. Furthermore, when looking at the global trends observed in the questionnaire data of the current study and related work, it appears that based on the context in which MAH feedback is applied, 
different types of benefits are observed. In our study, involving a home automation system, MAH feedback was perceived as a rather hedonic instead of utilitarian addition. Furthermore, no general benefits in terms of pragmatic UX were observed, which was similar to other studies outside of an automotive context [24][15] [5]. Only hedonic UX, engagement, and users' emotional responses were positively impacted by the addition of MAH feedback in the current study. Apart from the valence of the emotional response, all these benefits remained present over the course of five weeks, thus were probably not merely due to a novelty effect. Similarly, when considering an entertainment context, MAH feedback has shown higher engagement when added to interactive kiosks [9], and led to increased enjoyment when playing a virtual piano [27]. Thus, for devices developed in a neutral, home-related, or entertainment context, we would recommend adding MAH feedback because, although no pragmatic benefits were observed, users did experience more joy and pleasure while interacting, even after repeated use.

\section{B. Limitations and Future Research}

A first limitation of the current study is the absence of an elaborate pre-study in which the optimal combinations of MAHs with gestures were investigated. An optimal fit between MAHs and gestures could have led to different results with regard to usefulness and ease of use. A second limitation is the use of the finger tip as a location to render the MAH feedback. MAH feedback on the finger tip is harder to perceive than on the palm of the hand. This discrepancy in perceivability might explain the frustration that some participants experienced while interacting with the device. A third limitation is that we did not assess participants' emotional states. A negative emotional state could have led to more negative user evaluations. However, our sample size would have been rather small to statistically test for a relationship between emotional state (individual difference) and the outcome variables. With this study, we only performed a first step in identifying the novelty effect's presence, more extensive research is needed to develop a comprehensive tool. Similarly, more research is needed to elaborate on the interview data observed in the current study. These data hinted at large individual differences in the perception of hedonic and pragmatic UX qualities of MAH feedback and showed that it might be relevant to extend standardized questionnaires with more open ended, exploratory, and probing questions when trying to grasp "the complete UX picture". We thus suggest future research to take into consideration the nuance and possible ambiguity underlying statistical results, and to gauge these possibly hidden traits using a more qualitative approach. Furthermore, by relying on a larger sample size and/or more assessment points over time, one could statistically test for individual differences and how they change over time, as was done by Harbich and Hassenzahl [32].

\section{CONCLUSION}

The current study showed significantly increased enjoyment, engagement, and arousal of the emotional response when
MAH feedback was added to a home automation system, but during initial use and after repeated use. Furthermore, MAH feedback was perceived as a hedonic, instead of utilitarian, addition to the system. Although valence of the emotional response was elevated during initial encounter with $\mathrm{MAH}$ feedback, this effect disappeared after repeated use, showing the impact of the novelty effect. Interestingly, underneath the general tendencies observed in the questionnaire data, interview data hinted at potentially strong individual differences with regard to the perceived hedonic and pragmatic qualities of MAH feedback. Future research should consider systematically investigating individual differences in hedonic and pragmatic UX of MAH feedback.

\section{ACKNOWLEDGMENT}

We thank Jeroen Verfaillie and Bert Braeckman for programming the home automation system. This work was supported by the SHAKE project, realized in collaboration with imec. Project partners are Verhaert, Nokia Bell, NXP, imec and Mintlab (KU Leuven), with project support from VLAIO (Flanders Innovation and Entrepreneurship).

\section{REFERENCES}

[1] P. I. Cornelio Martinez, S. De Pirro, C. T. Vi, and S. Subramanian, "Agency in mid-air interfaces," in Proceedings of the 2017 CHI Conference on Human Factors in Computing Systems, 2017, pp. 2426-2439.

[2] E. Freeman, S. Brewster, and V. Lantz, "Tactile feedback for abovedevice gesture interfaces: Adding touch to touchless interactions," in Proceedings of the 16th International Conference on Multimodal Interaction, 2014, pp. 419-426.

[3] K. Harrington, D. R. Large, G. Burnett, and O. Georgiou, "Exploring the use of mid-air ultrasonic feedback to enhance automotive user interfaces," in Proceedings of the 10th International Conference on Automotive User Interfaces and Interactive Vehicular Applications, 2018, pp. 11-20.

[4] S. Brewster, F. Chohan, and L. Brown, "Tactile feedback for mobile interactions," in Proceedings of the SIGCHI conference on Human factors in computing systems, 2007, pp. 159-162.

[5] I. Rutten and D. Geerts, "Better because it's new: The impact of perceived novelty on the added value of mid-air haptic feedback," in Proceedings of the 2020 CHI Conference on Human Factors in Computing Systems, 2020.

[6] T. Iwamoto, M. Tatezono, and H. Shinoda, "Non-contact method for producing tactile sensation using airborne ultrasound," in International Conference on Human Haptic Sensing and Touch Enabled Computer Applications. Springer, 2008, pp. 504-513.

[7] R. Sodhi, I. Poupyrev, M. Glisson, and A. Israr, "Aireal: interactive tactile experiences in free air," ACM Transactions on Graphics (TOG), vol. 32, no. 4, pp. 1-10, 2013.

[8] G. Shakeri, J. H. Williamson, and S. Brewster, "May the force be with you: Ultrasound haptic feedback for mid-air gesture interaction in cars," in Proceedings of the 10th International Conference on Automotive User Interfaces and Interactive Vehicular Applications, 2018, pp. 1-10.

[9] H. Limerick, R. Hayden, D. Beattie, O. Georgiou, and J. Müller, "User engagement for mid-air haptic interactions with digital signage," in Proceedings of the 8th ACM International Symposium on Pervasive Displays, 2019, pp. 1-7.

[10] M. Koch, K. von Luck, J. Schwarzer, and S. Draheim, "The novelty effect in large display deployments-experiences and lessons-learned for evaluating prototypes," in Proceedings of 16th European Conference on Computer-Supported Cooperative Work-Exploratory Papers. European Society for Socially Embedded Technologies (EUSSET), 2018.

[11] D. Ablart, C. Velasco, and M. Obrist, "Integrating mid-air haptics into movie experiences," in Proceedings of the 2017 ACM International Conference on Interactive Experiences for TV and Online Video, 2017, pp. $77-84$.

[12] M. Hassenzahl, "The interplay of beauty, goodness, and usability in interactive products," Human-Computer Interaction, vol. 19, no. 4, pp. 319-349, 2004 
[13] D. A. Norman, "Cognitive engineering in user centered system design, da norman and sw draper, eds," Erlbaum, Hillsdale, $N J$, vol. 31, p. 62, 1986.

[14] S. Mahlke and M. Thüring, "Studying antecedents of emotional experiences in interactive contexts," in Proceedings of the SIGCHI conference on Human factors in computing systems, 2007, pp. 915-918.

[15] E. Maggioni, E. Agostinelli, and M. Obrist, "Measuring the added value of haptic feedback," in 2017 Ninth International Conference on Quality of Multimedia Experience (QoMEX). IEEE, 2017, pp. 1-6.

[16] F. D. Davis, "Perceived usefulness, perceived ease of use, and user acceptance of information technology," MIS quarterly, pp. 319-340, 1989.

[17] V. Venkatesh, M. G. Morris, G. B. Davis, and F. D. Davis, "User acceptance of information technology: Toward a unified view," MIS quarterly, pp. 425-478, 2003.

[18] D. B. Köse, B. Morschheuser, and J. Hamari, "Is it a tool or a toy? how user conceptions of a system's purpose affect their experience and use," International Journal of Information Management, vol. 49, pp. 461-474, 2019.

[19] H. Van der Heijden, "User acceptance of hedonic information systems," MIS quarterly, pp. 695-704, 2004.

[20] H. O'Brien, "Theoretical perspectives on user engagement," in Why Engagement Matters. Springer, 2016, pp. 1-26.

[21] H. L. O'Brien, P. Cairns, and M. Hall, "A practical approach to measuring user engagement with the refined user engagement scale (ues) and new ues short form," International Journal of Human-Computer Studies, vol. 112, pp. 28-39, 2018.

[22] M. Schrepp, A. Hinderks, and J. Thomaschewski, "Design and evaluation of a short version of the user experience questionnaire (ueq-s)." IJIMAI, vol. 4, no. 6, pp. 103-108, 2017.

[23] G. Korres, S. Chehabeddine, and M. Eid, "Mid-air tactile feedback colocated with virtual touchscreen improves dual-task performance," IEEE Transactions on Haptics, 2020.

[24] A. Sand, I. Rakkolainen, P. Isokoski, J. Kangas, R. Raisamo, and K. Palovuori, "Head-mounted display with mid-air tactile feedback," in Proceedings of the 21st ACM Symposium on Virtual Reality Software and Technology, 2015, pp. 51-58.

[25] M. Hassenzahl, "The thing and i: understanding the relationship between user and product," in Funology 2. Springer, 2018, pp. 301-313.

[26] V. Venkatesh, J. Y. Thong, and X. Xu, "Consumer acceptance and use of information technology: extending the unified theory of acceptance and use of technology," MIS quarterly, pp. 157-178, 2012.

[27] I. Hwang, H. Son, and J. R. Kim, "Airpiano: Enhancing music playing experience in virtual reality with mid-air haptic feedback," in 2017 IEEE World Haptics Conference (WHC). IEEE, 2017, pp. 213-218.

[28] M. M. Bradley and P. J. Lang, "Measuring emotion: the self-assessment manikin and the semantic differential," Journal of behavior therapy and experimental psychiatry, vol. 25, no. 1, pp. 49-59, 1994.

[29] A. Betella and P. F. Verschure, "The affective slider: A digital selfassessment scale for the measurement of human emotions," PloS one, vol. 11, no. 2, 2016.

[30] J. A. Russell, A. Weiss, and G. A. Mendelsohn, "Affect grid: a singleitem scale of pleasure and arousal." Journal of personality and social psychology, vol. 57, no. 3, p. 493, 1989.

[31] E. Karapanos, J. Zimmerman, J. Forlizzi, and J.-B. Martens, "User experience over time: an initial framework," in Proceedings of the SIGCHI conference on human factors in computing systems, 2009, pp. 729-738.

[32] S. Harbich and M. Hassenzahl, "User experience in the work domain: A longitudinal field study," Interacting with Computers, vol. 29, no. 3, pp. 306-324, 2017.

[33] S. Kujala and T. Miron-Shatz, "Emotions, experiences and usability in real-life mobile phone use," in Proceedings of the SIGCHI Conference on Human Factors in Computing Systems, 2013, pp. 1061-1070.

[34] M.-H. Huang, "Designing website attributes to induce experiential encounters," computers in Human Behavior, vol. 19, no. 4, pp. 425442,2003

[35] S. Gallacher, J. O'Connor, J. Bird, Y. Rogers, L. Capra, D. Harrison, and P. Marshall, "Mood squeezer: lightening up the workplace through playful and lightweight interactions," in Proceedings of the 18th ACM Conference on Computer Supported Cooperative Work \& Social Computing, 2015, pp. 891-902.

[36] W. R. Hazlewood, E. Stolterman, and K. Connelly, "Issues in evaluating ambient displays in the wild: two case studies," in Proceedings of the SIGCHI conference on Human factors in computing systems, 2011, pp. $877-886$.
[37] L. Van den Bogaert, D. Geerts, and I. Rutten, "Grasping the future: Identifying potential applications for mid-air haptics in the home," in Extended Abstracts of the 2019 CHI Conference on Human Factors in Computing Systems, 2019, pp. 1-6.

[38] I. Rutten, W. Frier, L. Van den Bogaert, and D. Geerts, "Invisible touch: How identifiable are mid-air haptic shapes?" in Extended Abstracts of the 2019 CHI Conference on Human Factors in Computing Systems, 2019.

[39] D. Hajas, D. Pittera, A. Nasce, O. Georgiou, and M. Obrist, "Mid-air haptic rendering of $2 \mathrm{~d}$ geometric shapes with a dynamic tactile pointer," IEEE Transactions on Haptics, vol. 13, no. 1, 2020.

[40] O. Turel, "Quitting the use of a habituated hedonic information system: a theoretical model and empirical examination of facebook users," European Journal of Information Systems, vol. 24, no. 4, pp. 431-446, 2015.

[41] H. L. O'brien and E. G. Toms, "Examining the generalizability of the user engagement scale (ues) in exploratory search," Information Processing \& Management, vol. 49, no. 5, pp. 1092-1107, 2013.

[42] M. A. Lawrence, ez: Easy Analysis and Visualization of Factorial Experiments, 2016, $\mathrm{r}$ package version 4.4-0. [Online]. Available: https://CRAN.R-project.org/package $=\mathrm{ez}$

[43] A. Field, J. Miles, and Z. Field, Discovering statistics using R. Sage publications, 2012.

[44] G. H. Lunney, "Using analysis of variance with a dichotomous dependent variable: An empirical study 1," Journal of educational measurement, vol. 7, no. 4, pp. 263-269, 1970.

[45] T. S. Donaldson, "Robustness of the f-test to errors of both kinds and the correlation between the numerator and denominator of the f-ratio," Journal of the American Statistical Association, vol. 63, no. 322, pp. 660-676, 1968.

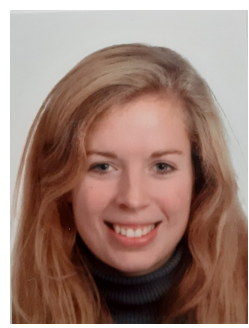

Isa Rutten started working at Mintlab after obtaining her PhD in Quantitative Psychology at KU Leuven in 2018. Intrigued by the way human cognition and novel technology interact with each other, she started working on the SHAKE project, focusing on quantitative assessments of the UX of mid-air haptic feedback.

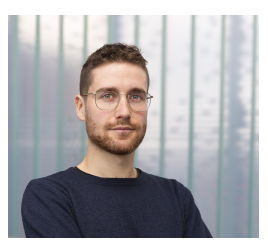

Lawrence Van den Bogaert Lawrence Van den Bogaert obtained a M.S. in Corporate Communications from the University of Leuven in 2014. After fulfilling both operational and commercial roles in industry until 2018, he turned back to the Social Sciences faculty at KU Leuven in search for a more research-driven position in an academic context. Since then, he is active as interaction researcher on different projects at Mintlab.

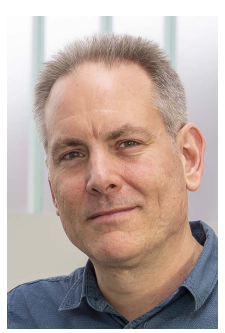

David Geerts "David Geerts is Research Manager of Mintlab at the Faculty of Social Sciences of KU Leuven. He is specialized in Human-Computer Interaction (HCI) and Human-Centered Design, more specifically in designing social interactions in online media and Social TV, and supervises several research projects in this domain." 